\title{
Digital Trends Survey 2019
}

\author{
Fabian Röthlisbergera, Reinhold Sojer \\ a collaborateur scientifique de la division Numérisation/eHealth de la FMH \\ ${ }^{\mathrm{b}}$ chef de la division Numérisation/eHealth de la FMH
}

\begin{abstract}
Dans les soins ambulatoires, la numérisation gagne continuellement en importance. Au-delà des développements dans le domaine de la documentation et de la communication numériques, les patients exigent davantage d'autonomie dans les processus décisionnels qui concernent leur propre santé.
\end{abstract}

Au fil des dernières années, un marché des applications s'est développé. Celles-ci permettent aux patients par exemple de contrôler de façon autonome des symptômes ou de surveiller leur état de santé sur la

\section{Les offres numériques sont toujours plus demandées par les patients.}

base de leurs propres données. A l'avenir, l'entretien direct avec les collaborateurs du cabinet médical, par exemple pour convenir d'un rendez-vous ou simplement obtenir des renseignements, devrait aussi être remplacé par des interactions avec des ordinateurs dotés de l'intelligence artificielle. Les patients s'attendent aussi de plus en plus souvent à des offres numériques, qui jouent un rôle important dans le choix d'un prestataire de santé. De plus, la disposition des patients à partager, sous certaines conditions, leurs données de santé avec leur médecin traitant augmente, ce qui permettra à l'avenir de soutenir une prise de décision participative par des applications numériques [1-3].

Mais quelles sont les applications numériques qui sont effectivement demandées par les médecins et la population suisse? Dans quels domaines les besoins des médecins diffèrent-ils de ceux de la population? Le Digital Trends Survey réalisé pour la première

\section{Démographie}

\begin{tabular}{lll} 
& Population & Corps médical \\
\hline Sexe & Hommes: $50,2 \%$ & Hommes: $57,5 \%$ \\
& Femmes: $49,6 \%$ & Femmes: $42,5 \%$ \\
& Sans réponse: $0,2 \%$ & Sans réponse: $0 \%$ \\
\hline Répartition par âge & $18-39: 34,2 \%$ & $18-39: 10,5 \%$ \\
& $40-64: 42,7 \%$ & $40-64: 81,6 \%$ \\
& $65+: 22,7 \%$ & $65+: 7,9 \%$ \\
& Sans réponse: $0,4 \%$ & Sans réponse: $0 \%$ \\
\hline
\end{tabular}

Figure 1: Répartition par âge et par sexe des échantillons. fois par la FMH veut répondre à ces questions. Le sondage s'oriente selon le concept du parcours du patient («Patient Journey») qui englobe tous les évènements vécus par le patient avant, pendant et après une consultation médicale. Le sondage de cette année met l'accent sur les applications qui promettent un appui numérique des processus avant la consultation médicale. Les offres analysées comprennent par exemple les options numériques pour une prise de rendez-vous au cabinet médical ou pour une première évaluation des symptômes. Dans les années suivantes, le Digital Trends Survey observera le soutien numérique de processus pendant et après la consultation médicale ainsi que la prévention.

\section{Méthode}

Un sondage en ligne a été réalisé en octobre et novembre 2019 en Suisse auprès des médecins travaillant dans le domaine ambulatoire et de la population âgée

\section{Le sondage a eu lieu en octobre et novembre} 2019.

de 18 ans et plus. La méthode et le questionnaire ont été développés conjointement avec des médecins, $\mathrm{PwC}$ Suisse et gfs.bern. La validité du questionnaire a été vérifiée au moyen de pré-tests cognitifs et quantitatifs. En plus des questions établies avec une échelle de Likert, le questionnaire comprenait une expérience de prise de décision qui a permis de réaliser une analyse conjointe. La méthode de l'analyse conjointe est un procédé multivarié qui mesure l'évaluation globale d'une prestation au moyen d'une compilation de différentes propriétés. Pour cela, on mesure quelle propriété est particulièrement importante pour l'évaluation globale de la prestation. Les personnes interrogées devaient choisir entre deux cabinets médicaux présen- 


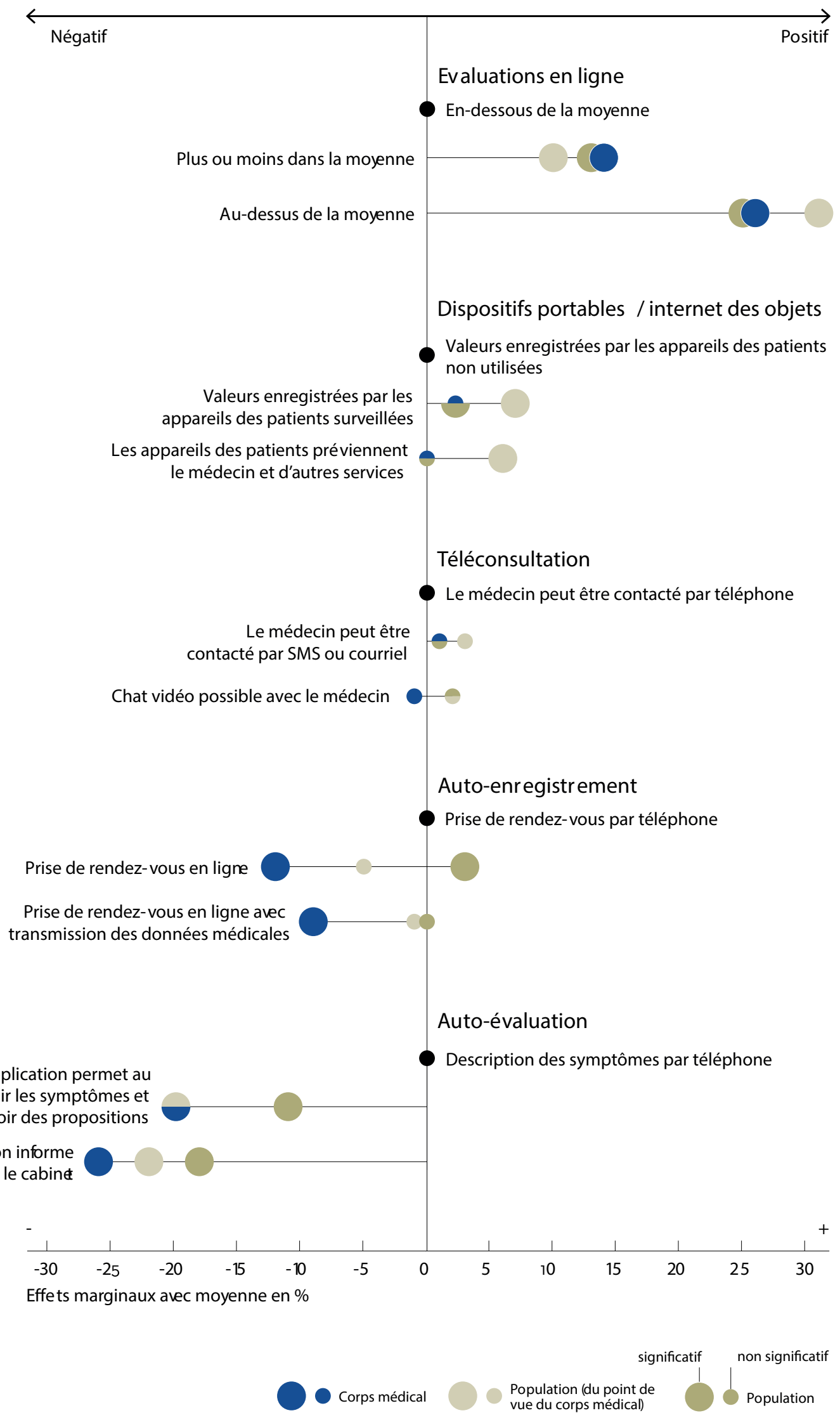

Figure 2: Evaluation de la plus-value de prestations numériques avant la consultation médicale. Plus les points tendent vers la droite/vers la gauche, plus la plus-value de l'offre numérique est jugée importante/faible par rapport à la valeur 0 . Pour chaque thème, la valeur 0 représente l'état avec le degré de numérisation le plus faible. 
tant des degrés différents de numérisation pour plusieurs dimensions [1].

La population était priée de désigner le cabinet qu'elle choisirait pour un traitement.

Les médecins devaient s'imaginer planifier un nouveau cabinet médical et indiquer quel cabinet ils considéraient comme plus adapté. De plus, ils devaient indiquer quel cabinet les patients choisiraient. La population était priée de désigner le cabinet qu'elle choisirait pour un traitement.

\section{Résultats du sondage}

494 médecins travaillant dans le domaine ambulatoire et 2432 habitant-e-s ont participé au sondage. La figure 1 décrit la répartition par âge et par sexe des deux échantillons.

\section{Les prestations numériques avant la consultation médicale}

Les résultats de l'expérience de prise de décision montrent que les offres numériques peuvent jouer un rôle déterminant lors du choix d'un cabinet médical (fig. 2). Alors que les médecins voient dans la prise de rendez-vous en ligne une perte en termes d'utilité (-12\%), la population considère que cette offre présente un gain d'utilité (+3\%). La population s'attend par contre à une plus-value supplémentaire marginale si les fonctions corporelles et paramètres vitaux saisis par le patient peuvent être surveillés et interprétés par le cabinet médical. Tant du point de vue de la population que de celui des médecins, les applications telles que SMS, courriel ou chat vidéo n'apportent pas de plus-value par rapport à la prise de contact téléphonique. Les deux groupes

\section{Alors que les médecins voient dans la prise de rendez-vous en ligne une perte en termes d'utilité, la population considère que cette offre présente un gain d'utilité.}

sont d'avis que les applications pour l'auto-triage se traduisent même par une perte en termes d'utilité et accordent la préférence à un cabinet médical qui obtient un score supérieur à la moyenne dans l'évaluation par rapport à un cabinet médical moins bien noté.

\section{Les intérêts de la population ne sont pas (encore) satisfaits}

Tant la population que les médecins estiment que l'utilisation des moyens numériques est importante pour l'approvisionnement en soins en Suisse. Globalement, l'intérêt de la population pour les offres numériques est plus élevé que celui des médecins. Alors que les médecins manifestent le plus grand intérêt pour les ouvrages de référence en ligne (48\%), la population s'intéresse notamment à la notification automatique des rendez-vous ou des temps d'attente (89\%). Comparativement à d'autres branches, le système de santé n'est que peu numérisé: alors que la plupart des personnes $(87 \%)$ règlent leurs opérations bancaires et leur déclaration d'impôt (79\%) par voie numérique, le contact avec les professionnels de la santé se déroule le plus souvent par voie analogique (87\%). Même si une grande partie du corps médical connaît beaucoup d'applications numériques, seule une minorité propose de telles prestations au cabinet. La prestation la plus souvent proposée (32\%) est l'envoi de photos au médecin par le biais du smartphone. Une majorité des médecins (79\%) connaît les offres pour la prise de rendez-vous en ligne, mais seule une minorité $(10 \%)$ en fait usage au quotidien. Les médecins s'attendent en majorité (92\%) à de nouveaux problèmes de protection des données et sont d'avis que tant les patients (88\%) que les médecins (86\%) doivent être davantage sensibilisés à la manière de gérer les données de santé numériques.

\section{Satisfaction élevée à l'égard des médecins}

Le degré de numérisation du cabinet médical joue un rôle secondaire pour ce qui concerne la satisfaction de la population par rapport aux prestations médicales. Alors que la population évalue diversement l'offre numérique actuelle des médecins ou que beaucoup d'habitant-e-s estiment ne pas (encore) pouvoir l'évaluer, la grande majorité est satisfaite des prestations médicales (médecins de famille: $86 \%$, spécialistes: $73 \%$ ) et près de trois quarts de la population recommanderait son médecin à un ami. De plus, le fait que le médecin participe au dossier électronique du patient (DEP) ou non ne joue pratiquement aucun rôle: seule une minorité de la population (15\%) attache une telle importance au DEP qu'elle serait prête à changer de médecin si celui-ci n'y participe pas.

\section{Discussion et perspectives}

A l'heure actuelle, les médecins utilisent les outils numériques "avant le traitement» avec retenue. Les raisons de cette réticence sont expliquées dans la littérature par l'absence d'interopérabilité et une preuve de l'utilité insuffisante [4]. Les résultats de ce sondage permettent aussi de conclure à la nécessité de mesures de sensibilisation en matière de protection des données, 
tant pour les médecins que pour la population. Grâce aux aides proposées, par exemple les «Exigences minimales pour la sécurité informatique» [2], la FMH permet aux médecins de garantir une protection des données appropriée. Parallèlement, il faut demander à

Alors que la numérisation était jusqu'ici «facultative" pour les cabinets médicaux, la situation actuelle crée une certaine pression.

l'industrie de créer des solutions aussi conformes que possible aux exigences en matière de protection des données.

Malgré les craintes liées à la protection des données, les médecins doivent comprendre que les offres numériques seront à l'avenir de plus en plus exigées par leurs patients et qu'elles pourraient devenir un critère déterminant dans le choix du médecin. La pandémie actuelle de COVID-19 peut aussi être une chance: alors que la numérisation était jusqu'ici «facultative» pour les cabinets médicaux, la situation actuelle crée une certaine pression en faveur de l'utilisation des applications numériques pour réduire le contact direct avec les patients et donc un éventuel risque de transmission. Quant à savoir si les outils utilisés à cet effet s'imposeront une fois la pandémie passée, cela dépendra de l'utilité supplémentaire tangible pour les médecins et des incitations politiques dans ce sens.

Le rapport complet relatif au Digital Trends Survey, y compris l'évaluation des résultats par des experts, peut être consulté sur le site internet de la FMH: www.fmh.ch/ digital-trends-survey-2019-fr

\section{Références}

1 S. A. Rahimi, M. Menear, H. Robitaille, and F. Légaré, "Are mobile health applications useful for supporting shared decision making in diagnostic and treatment decisions?", Glob. Health Action, 2017.

2 M. Bradway et al., "Measuring the Effects of Sharing Mobile Health Data During Diabetes Consultations: Protocol for a Mixed Method Study", JMIR Res. Protoc., 2020.

3 E. Seltzer et al., "Patients' willingness to share digital health and non-health data for research: A cross-sectional study", BMC Med. Inform. Decis. Mak., 2019.

4 T. Kong, M. M. Scott, Y. Li, and C. Wichelman, "Physician attitudes towards - and adoption of - mobile health", Digit. Heal., 2020.

5 Pour l'expérience de prise de décision, les cinq dimensions suivantes des offres numériques d'un cabinet médical ont été analysées: auto-évaluation, télémédecine, auto-enregistrement, évaluations en ligne et dispositifs portables/internet des objets médicaux.

6 https://www.fmh.ch/fr/prestations/ehealth/securite-informatique.cfm

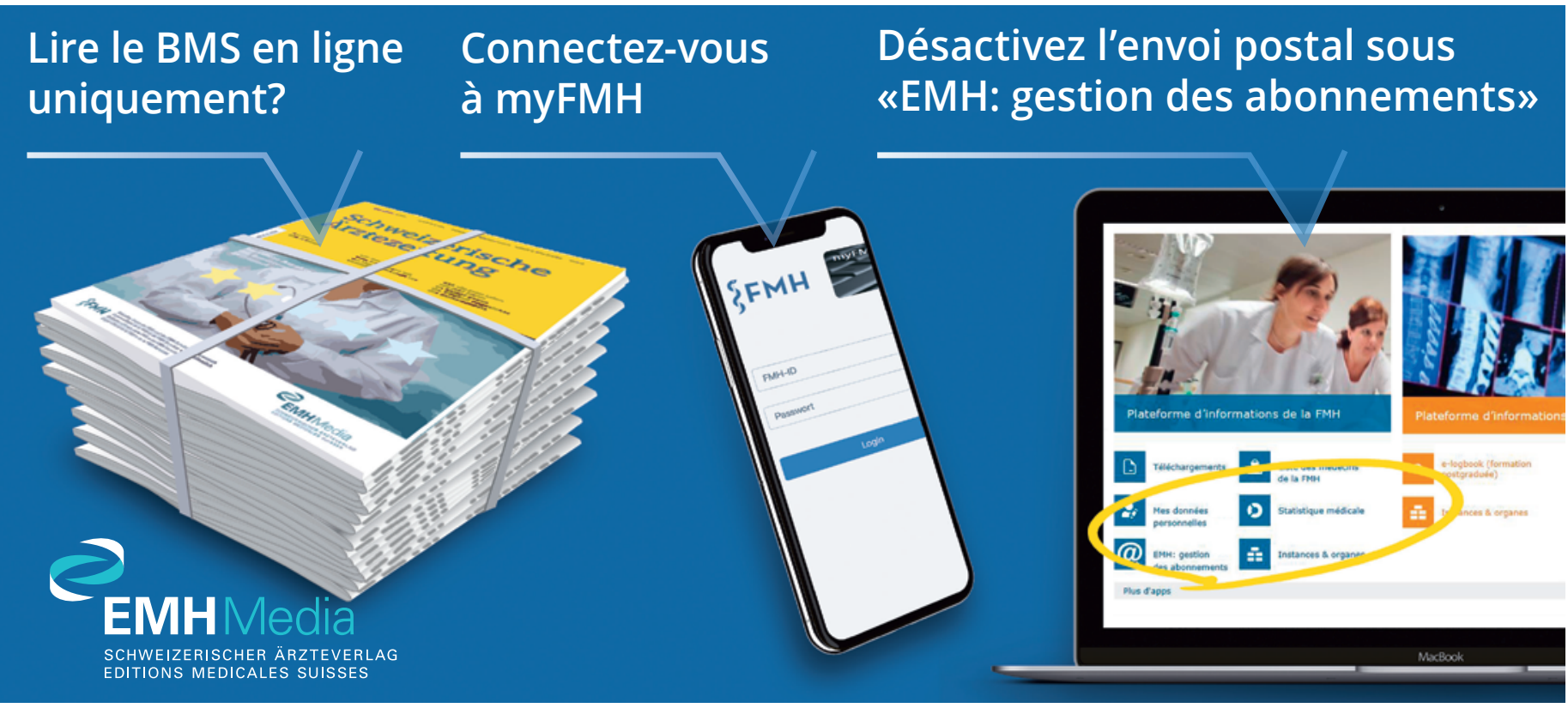

